ne porterait pas préjudice aux poissons qui s'accommodent parfaitement d'un certain degré de salure des eaux ; elle leur serait, au contraire, trés favorable en leur fournissant un appoint de nourriture sous furme de plancton qui pullule dans les eđux légèrement salées. De plus, cette pratique provoquerait la pénétration en Brière de poissons littoraux tels que les Muges, Flets, eto., qui en augmenteraient, d'une façon appréciable, les ressources piscicoles.

Les canaux creusés pour l'asséchement formeraient des retraites profondes où le poisson pourrait se réfugier, en hiver pour se mettre â l'abri du froid et, en été, pour s'abriter de la chaleur.

Les joncs et roseaux qui ne manqueraient pas do pousser avec vigueur dans ces eaux peu profondes, seraient coupés chaque année par des bateaux faucardeurs dont il existe maintenant d'excellents modèles et on pourrait, peut-être, chercher $\dot{u}$ les utiliser pour la fabrication de la cellulose.

Quant à la tourbe dont les habitanls font leur combustible hahituel et qui est, pour eux, une ressource importante, son extraction pourrait être faite économiquement par des dragues spéciales qui la leur foumiraient à un prix bien inférieur à celui auquel elle leur revient actuellement, si l'on tient compte de la main-d'ouvie qu'ils y consacrent. On pourrait môme on vendre alı dehors le jour où la tourbe serait utilisée industriellement.

En résumé, l'asséchement de la Brière ne paralt présenter qu'un intérêt économique très douteux et, par contre, son maintien constant en eau, que permettraient de réaliser les ouvrages construits pour l'asséchement, semblerait devoir offrir de très gros avantages.

Il y aurait donc lieu de poser nettement la question devant les autorités compétentes, en l'envisageant sous son double aspect, et d'examiner sí la ligne de conduite suivie jusqu'ici ne devrait pas être modifiée.

\title{
LA PRODUCTION DE LA FEUILLE
}

\author{
Par le Comte de NEUfBourg \\ Prèsident du Syndicat des propriétaires et exploitants \\ des étangs du Porez
}

On n'en peut plus douter : la Carpe cuir de 3 livres se vend deux fois plus cher et beaucoup plus facilement que ne se vend la Carpe commune d'une livre.

D'autre part, cette Carpe sélectionnée arrive à ce poids de 3 livres très facilement en 3 étés, parfois en 2 étés, c'est-à-dire qu'elle rend 3 à 4 fois plus que ne fait la Carpe commune dans le même temps.

Enfin, si votre étang produit ion kilos à l'hectare, il vous faut, pour 
les obtenir, immerger 200 tètes de nourrain commun de t5o grammes, soit 3o kilos d'empoissonnage, pour n'être pas sủr de récolter 100 kilos de Carpes d’une livre. Aver des Cuir sélectionnées, en immergeant 66 têtes de nourrain de 250 grammes, soit I $6 \mathrm{kil}$. 5oo, vous obtenez vos roo kilos de belles Carpes : autre économie de transport et de manutention, sans surcroît de dépense, en payant le bon nourrain le double du mauvais.

Notez encore que, si vous déjoncez, hersez, chaulez, fumez votre étang, la cuir profitera davantage de ces frais que ne pourra faire la commune abâtardie.

Additionnez ces quatre causes de profits, et, si rous l'osez, remettez à l'eau vos harengs.

Il est donc évident que tout le monde a l'occasion de quintupler son bénéfice en utilisant de l'empoissonnage sélectionné. La difficulté est de trouver cet empoissonnage.

Le nourrain cuir choisi n'est pas cher s'il est payé le double du commuII, mais le second existe sur place et le premier se transporte. Or, les transports, s'ils ne peuvent se faire en camion, sont très chers. Beaucoup d'emboucheurs de Carpes préfèrent donc faire venir une feuille de 30 grammes plutôt qu'un nourrain de 300 grammes. Observez que le coùt n'en est pas yo fois moindre, car il faut 2 fois plus d'eau pour transporter loin une tonne de feuilles qu'il n'en faut pour une tonne de nourrains. Il y a pourtant une économie, puisquau lieu d'une tonne de nourrains on ne fait voyager que 100 kilos de feuilles. Cette économie s'augmente encore du fait qu'au lieu d'acheter I kilo de nourrain, par exemple à 8 francs, on n achète cque 100 grammes de feuille, à $\mathbf{6} 6$ francs le kilo, soit I fr. 60 .

Il est vrai que, si l'étang niest pas assez bon pour donner du 2 étés de 2 livres marchand, on atlend un an de plus l'intérêt de son argent.

Cependant, ces diverses raisons font que les engraisseurs de Carpes, non oulillés pour produire l'alevin avec fruit, sécurité et garantie, tendent à rechercher la feuille plutôt que le nourrain.

On constate, même, que la demande irançaise rejette la belle feuille de plus de 50 grammes comme trop coûteuse el préfère celle de 20 à 30 grammes, voire de io à 15 grammes. L'économie initiale est incontestable ; de bons praticiens la pensent trompeuse. Sur leurs conseils, j'emploie ma feuille de 5o ou de 200 grammes (on en trouve, certaines années, de 35o) à produire du 2 étés de 2 à 3 livres et je m'en trouve bien. Mais j'avcue que la feuille de 20 grammes, immergée en nombre convenable, arrive à produire souvent le rendement complet de l'étang en atteignant 2 livres. Nous en avons plusicurs exemples : un étang produisant 200 kilos à l'hectare avait reçu joo têtes de feuilles de $\mathbf{z}$ grammes destinées à donner des nourrains de foo érammes, pour satisfaire à une demande de ce poids. Or, les llérons et Cormorans aidant, et l'année se trouvant très bonne, l'étang ne rendit que 250 têtes, mais de 900 à 
I.100 grammes. Le poids total prévu était dépassé, mais la commande ne put être satisfaite et le nourrain fut expédié aux Halles où il se vendit $\hat{y}_{0} \%$ de moins qu il n'était vendu comme empoissonnage.

Une autre fois, nous avions laissé, par erreur, dans un trou de botte, quelques feuilles de Septemlire de 2 à 3 grammes, que l'on compta le lendemain, alors qu'elles inspectaient les rives de l'eau montante dans le petit étang reformé : il y en avait 48. Le bassin resta inutilisé : 8 mois après, on y trouva 40 nourrains faisant 39 kilogrammes. Certaines feuilles avaient pris 333 fois leur poids inilial !

Je ne prendrai donc pas parti dans la querelle : feuilles de 5o grammes ou de 20 grammes, toutes sont uliles si elles sont racées. Les grosses peuvent faire du 2 étés bon marchand, les autres du très beau nourrain. Certains assurent que la petite feuille se transporte mieux que la grosse ; ce me semble être une erreur d'observation incomplète.

D'ailleurs, un groupe de géniteurs donne ces deux tailles de feuilles normalement.

La pose de Mai dépasse aisément 5o grammes, celle d'Août demeure entre 20 et $3 o$ grammes. Bien entendu, tout dépend de ce que les nouveau-nés trouvent à manger : au large et richement alimentés, la première pose ne s'arrêtera qu'entre 200 et 300 grammes, sans empêcher le second lit d'arriver à 5o grammes, mais le temps lui manque pour franchir celte limite. Enfun, les poses peuvent se produire en Mai et Juillet, ou bien en Juin et Août, parfois en Septembre, au gré du temps. Il est rare que la pose de Mai soit seule, mais fréquent que celle de Juillet, même première de l'année tardive, n'ait pas de suite. Les feuilles sont alors assez uniformes et leur poids varie à peine du simple au double. Si l'on veut détruire une pose tardive, on peut introduire quelques lancerons très petits, en fin d'Août : mais la difficulté est de conserver ces lancerons tout l'été sans les laisser grossir.

La frayère hors l'étang permet, en principe, de régler le poids des feuilles selon qu'on en lâche plus ou moins à l'étang de premier été. Mais les erreurs d'appréciation sont faciles avec ces petits êtres sujets à tant de hasards, et l'on ne fait pas sur commande de la feuille d'un poids donné comme on arrive à faire du nourrain. Il y a donc un triage nécessaire après pêche. Voilà le difficile : car le tripotage est mortel aux feuilles et vous risquez de recevoir 1 .ono têtes vivantes pour n'en retrouver que 5oo un mois après, surtout s'il s'agit d'écailleuses. Il faut donc laisser les feuilles se séparer elles-mîmes en grosses et petites. Toutes les combinaisons sont possibles sur ce thème : les alevins déposés en aval du courant remonteront en amont, en passant au travers des barreaux d'une grille de bois doux dont on règle l'éloignement selon le triage qu'on veut opérer. Au bout d'un temps variable, la petite feuille a gagné l'amont, la grossè est restée le nez aux barreaux. Bien entendu l'opération n'est pas aussi simple, mais son détail est commandé par la disposition des lieux : il suffit que la grille soit large pour éviter la bous. 
culade et que la mancuvre soit arrêtée dès qu'elle ne donne plus rien d'utile. On peut aussi laisser la feuille reposer dans une pantenne ou filet de maille voulue : seule la petite feuille s'échappera.

S'il faut trier quelques sujets ou des étrangers comme la Perche, la Tanche, le Chat, on peut pecher la feuille au filet sans nceuds ; la porter dans des bassines, sans trous où elle puisse s'engager ; la vider sur la table de triage tenue à plat et le bec bouché d'un torchon. La feuille alage dans un pouce d'eau : on enlève à la main les indésirables qu'on distingue fort bien, puis on débouche le bec, on soulève l'opposé de la table et les feuilles coulent dans le bassin saus avoir l'air ni la main.

Venons-en au point le plus important.

La feuille n'est demandée qu'en Janvier et jusqu'en Mars. Or, il ne faut la pêcher que peu de jours avant de l'expédier, car elle souffre en dépôt, y perd rapidement 20 à $25 \%$ de son poids, s'y écaille et donne un manque de têtes d'autant plus grrand, dans l'étang de deuxième été, (qu'elle cst restée plus longtemps en dépót. En Pologne, en Allemagne, on stabulait la feuille très soigneusement d'Octobre à Mars pour lui éviter les surprises du gel, les dangers hivernaux à l'étang. Ces dépôts sont admirablement conçus et surveillés ; l'eau y coule abondamment ; la glace y est rendue inoffensive par des bâtis tranversaux. La feuille n y entre que désinfectée, débarrassée des parasites et nettoyée au bain de sel ou d'ammoniaque. Cependant on abandonne ces procédés : on a moins de pertes de têtes en transportant au plus vite la feuille de son ćtang de premier été à celui de deuxième été.

Cièa tient à ce que la feuille n'est pas de mocurs identiques à celles de la Carpe adulte. File ne vit pas aussi facilement sur sa graisse, en hiver, que la Carpe dont la croissance est, sinon achevée, au moins ralentic. L'extraordinaire plasticité de l'alevin, encore considérable dans son deuxième été, l'oblige à prendre quelque nourriture en hiver dès que la température de l'eau de surface le permet et qüil peut visiter les rives vite réchauffées, vite peuplées d'insectes, où $n$ 'atteint pas la Carpe de 3 pouces de haut, qui, elle, ne quitte pas volontiers les fonds à tempéralure uniforme, encore que cela lui arrive dans les jours mous de l'hiver, comme en Mars 19:8. Il est certain que des feuilles immergées rans leur étang de deuxième été en Novembre 19?7, alors qu'elles pesaient is grammes, faisaient 30 grammes en Mars et 40 en Avril : l'étang avait été à sec l'année précédente ct ses rives étaient déjoncées par le hétail. L'alevin avait travaillé, à la faveur de quelques rayons de soleil et du vent du sud.

Le dépòt exclu, l'éleveur se trouve fort embarrassé. Devra-t-il attendre Février ou Mars pour vider ses ćtangs de premier été, les nettoyer, herser, chauler, fumer, les préparer à la ponte suivante? Il ne pourrait alors compter faire produire ces étangs de pose qu'un an sur deux. En fait, c'est souvent le cas et ce n'est pas perte sèche, car la pose est meilléure .dans un étang aéré toute une année. Mais l'assec n'est pas absolument 
nécessaire. En effet, si la feuille mange en hiver, c'est fort peu relativement à sa gloutonnerie estivale. On peut donc doubler, tripler la jeune population d'un étang de Novembre à Mars, sans nuire à la santé de la feuille. On pêchera donc avec grrand soin, par temps couvert et sans glace, un étang de pose dont le fond, devant la bonde, aura été entretenu exempt de vase et dont le trou d'arrière-bonde, à la sortie du chéneau de bois, sera planchéié et nettoyé. Les filets fins et sans nœuds seront maniés doucement et vidés dans des bidons remplis à l'avance d'un tiers d'eau propre et de température égale à celle de l'étang. Ces bidons seront portés aussitôt à l'ćtang le plus voisin, contenant une pose de même sorte et qui ne sera pêché que la veille de l'expédition.

Si les deux étangs se touchent, on pourra, si le terrain s'y prête, établir une communication, un courant entre eux : en chassant un peu la feuille de l'étang inférieur vers ce courant, on pourra en faire remonter une

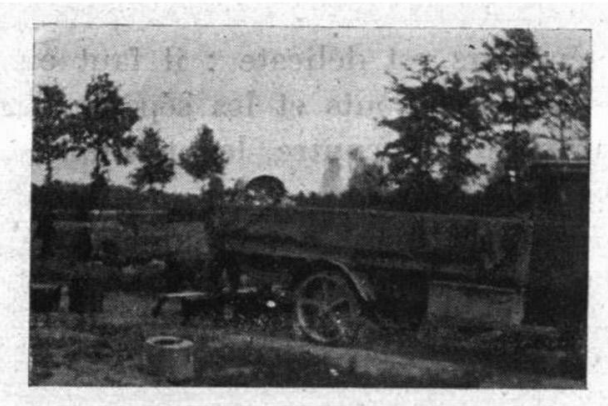

1. Chargement d'un camion ì l'étang.

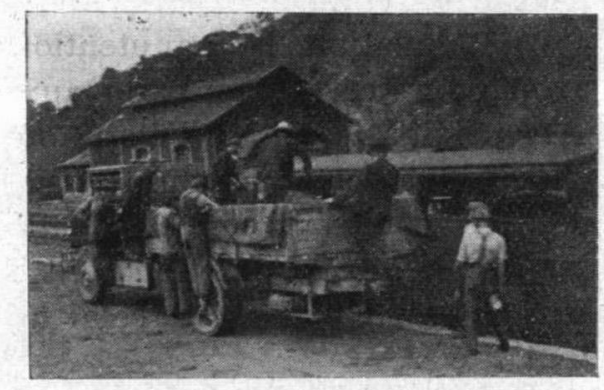

¿. Chargement d'un wagon en gare.

grande partic; dans l'étang supérieur. Ce sera autant de sauvé de tout risque.

La livraison par grande quantité, sur wagon, se fera à jour convenable, couvert ef sans gel. Iń dépêché préviendra l'acquéreur assez tôt pour qu'il puisse exécuter les mesures prévues pour l'arrivée. Le wagon peut lâcher partie de son chargement 'on cours de route, à un co-acquéreur. Mais la livraison échelonnéc n'est facile qu'en camion bâché et muni d'oxygène. C'est le mode de l'avenir et les progrès de l'empoissonnement n'en seront que plus rapides et plus sûrs.

Si l'on juge l'emploi de l'oxygène susceptible de troubler l'alevin, de l'enivrer, on peut employer la pompe à moteur qui aère l'eau sans diffusion de gaz. M. Peupion met au point une pompe transportable fort intéressante à cet égard.

Le camion permet la livraison syndicale ; l'organisation en est très facile : plusieurs régions la pratiquent avec fruit.

Quels risques court le producteur de feuilles en faisant poser de nombreux étangs, petits ou moyens, au licu d'y produire du nourrain ou du marchand? 
Dabord, la pose, même avec le secours des Dubich, peut manquer totalement au printemps, ce qui réduit beaucoup le poids total rendu par l'étang et ne donne que de la petite feuille d'été, suivie peut-être de feuilles tardives qui mangent, mais sont perdues à la pêche en raison de leur taille minuscule et de leur fragilité.

Ensuite le pourcentage de perte à la peche est plus élevé pour les feuilles que pour les nourrains, avec lesquels il doit tendre à zéro. Les frais de pèche sont élevés, en raison des soins nécessaires et du choix d'un personnel éduqué. Préparation de l'étang, évacuation de la vase, iménagement du sol, ansendements, surveillance en cours d'année, occasionnent des débour's supplémentaires.

Les étangs pêchés tardivement seront souvent inutilisables un an sur deux, ou, mal remplis, ne recevront qu'une femelle au lieu de deux et ne rendront que demi-produit ; il est vrai que, dans ce cas, un remplissage tardif, s'il ne noie pas le frai, donne d'excellents résultats et qu'il peut être temps de rajouter des géniteurs.

La stabulation, la manutention des génitcurs est délicate : il faut en avoir en réserve pour parer aux pertes, aux accidents et les séparer de sexe jusquìa la mise à l'étanđr ou à la frayère Par contre, la production d'alevinage demande peu de frais d'empoissonnage, encore qu'un groupe de bons géniteurs contrôlés vaille son pesant d'écus. Notons aussi que, lorsque le rendement est hon, le poids tolal donné par un étang en feuilles arrive à dépasser celui qu il peut fournir en Carpes de 2 ou surtout 3 étés.

Yéanmoins, il serait absurde de consarrer 20 hectares d'eau à la pose, d'immerger 40 on 50 groupes laborieusement choisis et conduits depuis 4 ou 5 ans, sans être sûr d'ócouler la leuile à venir.

Si l'on veut réduire les priv de revicul, donc de vente, il faut réduire les risques du producteur : la leuille doit donc être commandée, vendue, avant la mise à l'cau des quéniteurs, c'l fait avant la Noël, soit ı 3 à 14 mois avant sa livraison. I e producteur doit pouvoir réduire la livraison de $\overline{5} \% \%$, car il serait incapable de fournir une marchandise honnête si la nature la lui a refusée.

Dans res conditions, la production de la feuille peut se faire à bon compte pour le vendeur of l'acyúreur. I'ne feuille incertaine, inconnue, occasionnelle est toujours chère. I we feuille dont vous avez vu mettre à l'eau les génileurs, vu la pîche el le chargrement, ce qui vous est facile, n'est jamais rhère. Celte question de contrôle est primordiale, tout le monde le comprend.

Si l'on veut avoir notion des quantités d'empoissonnage nécessaires à la mise en valeur des étangs français, comptons qu'il y a 100.000 hectares exploitables. Estimons à $9^{(0.000}$ hectares l'étendue à consacrer au poisson marchand de 3 étés, qui pourrait produire 9 millions de kilogrammes très facilement. Il est donc nécessaire, pour récolter ces 9 millions de $\mathrm{kgs}$, d'immerger a à 3 millions de kgs de nourrains, qui doivent être pro- 
duits sur des étangs à rendement supérieur, afin que la sélection soit convenahle. Donc ıo à İ̈.ooo hedtares seront nécessaires à la production du nourrain destiné à multiplier au moins ; fois son poids. Pour avoir res 3 millions de hilogramnes de nourrains, il laut trouver 300.000 kilogrammes de feuilles capables de donmer ro fois leur poids; il suffira d'affecter $1 . \bar{j}$ oo hectares à la poute. Or, à cette heure, nous avons 20.000 hectares empoissonnés un peu rógulièrcment el il ne se fait pas ro.ooo kilogrammes de feuilles sélectionnées et 15.000 de nourrains.

On voit qu'il y a de la marge pour la production de l'alevin.

Nous ne dirons rien de la pratique, du métier du producteur : c'est un autre chapitre. Nous voulions seulement exposer les avantages de l'acquéreur d'empoissonnage sélectionné et montrer que la production de cet empoissonnage dépend de l'organisation de la commande. Vous en avez déjà tiré les conclusions.

\title{
LE FÉTICHISME DE L'ÉTIQUETTE
}

\author{
Par M. Ritoul de IDROUIN de BOUVILLE
}

M. Luérituen est un émule de Bollesu : il appelle un chat un chat et souhaiterait que la mode s'en répandît (1).

Non seulement il se refuse à prendre les vessies pour des lanternes, mais il prend plaisir à les dénononfler par de malicieuses piqùres.

Champion de la clarté, de l'ordre et du bon sens il ne craint pas d'émouvoir des candeurs ou de déranger des combines. Ne s'est-il pas avisé, récemment, de réclamer une rectification d'élat civil pour la Perche-Truite? plus et mieux de confester le droit des Carpes gauloises à se parer de titres inscrits au Gotha germanique?

Sur le premier chef du réquisitoire, concédons que la dénomination couramment attribuée au Microptère salmoüde induit en erreur les profanes. Ne le voient-ils pas se présentant ì la façon de l' "Amour moderne ":

\footnotetext{
Je suis le fruit d'un rendez-vous

Pris dans un paol, en Amérique,

Entre une Truitelle a points roux

Et une Perche chlorolicjue!
}

Or, il ne s'agit aucuncment d'un métis ; ce citojen des Elats-Tnis appartient à une honorable famille: celle des Centrarchides, est de bonne vie et mœurs et on ne lui connaît pas de liaisons, comme à la Brême, au Gardon et autres Cyprins de petite vertu. C'est lui faire tort

(1) Voir Bulletin de septembre, p. 5r. 